\title{
Application of Superposition Coding for Subcarrier and Bit Allocation in Downlink OFDM Systems
}

\author{
Karan Nathwani, Member, IACSIT, Aditya Trivedi, and Kalpana Goyal, Member, IACSIT
}

\begin{abstract}
Orthogonal frequency division multiplexing (OFDM) is well known for multicarrier modulation technique in which a high data rate stream is divided into different parallel lower data stream. In this paper, we study the margin adaptive - superposition coding (MA-SC) algorithm and rate adaptive - superposition coding (RA-SC) algorithm, where at most two user can share each subcarrier as compared to MA and RA algorithm, where each subcarrier is shared by only single user without SC scheme. We apply SC scheme over MA and RA to achieve maximum system throughput with separate power constraint for real and non real time users, ensuring that QoS requirement for real time and proportional fairness among non real time users is satisfied. The overall computational complexity of proposed modified RA-SC algorithm is same as RA algorithm. In the modified proposed MA-SC, complexity increases in some of the steps due to addition of SC scheme over MA algorithm, but overall complexity remains the same. Monte-Carlo simulations are being carried out to show that the proposed algorithm improves the performance in terms of power required and throughput.
\end{abstract}

Index Terms-MA-SC, OFDM, RA-SC.

\section{INTRODUCTION}

Orthogonal frequency division multiplexing (OFDM) is an efficient technique for providing high data rate system and its ability to increase the symbol time large enough so that the induced delays produced by channel are only insignificant portion of the symbol duration, which makes OFDM system capable of reducing or eliminating intersymbol interference (ISI) [1].

Resource allocation are generally of two types-first fixed or static resource allocation, where we do not utilize the knowledge of channel state information (CSI) and users have predetermined knowledge of subcarriers on which they can transmit. Another one is dynamic resource allocation, where each user take advantage of CSI in selecting the best subcarrier for them. Again based on different optimization problem, resource allocation can be divided in two parts-first is margin adaptive (MA) optimization for real time users discussed in [2]-[6], which minimizes the overall transmit power assuring that bit error rate (BER) and bit rate requirements is satisfied, second is rate adaptive (RA)

Manuscript received January 28, 2012; revised March 5, 2012. This work was supported by ABV-IIITM Gwalior.

Karan Nathwani is with the Indian Institue of Technology, Kanpur. (e-mail: karan046@gmail.com).

Aditya Trivedi is with the Department of ICT, ABV-Indian Institute of Information Technology and Management, Gwalior, India (e-mail: atrivedi@iiitm.ac.in).

Kalpana Goyal is with the Department of Electronics, Maulana Azad National Institue of Technology, Bhopal, India (e-mail: kalpana.goyal19@gmail.com). optimization algorithm for non-realtime users discussed in [8]-[11], with a principle of maximizing the system throughput having constraints of overall transmit power, proportional fairness and BER requirement.

Both MA for real time user and RA for non real time user is considered in paper [7], which utilizes heterogeneous services to maximize the overall system throughput under the total transmit power constraint making sure that the proportional fairness among non-realtime users and QoS requirement of real time users is satisfied. In [8], in order to achieve the effective capacity for multicast transmission SC technique is used without taking multiuser access technique into account. Simple watermarking system is proposed in [9], which allows two classes to be transmitted on the same channel with the same frequency plan.

A suboptimal algorithm with an objective to achieve maximum system throughput is discussed in [10] with traffic requirement of users. SC is used in [11] to perform subcarrier, bit and power allocation by allowing at most two users to share same subcarrier. But they have applied this scheme for only real time users without taking non real time users into account. In [11] and [12], author first allocate a subcarrier to a weakest link or far user and allow these subcarrier to be shared by a potential user (near to the BS) using SC scheme. In [6], resource allocation algorithm without integer bit constraint for heterogeneous services in practical adaptive OFDMA systems is considered, which is further investigated in [7] for adaptive resource allocation problem in OFDMA systems with heterogeneous services.

In this paper, we extend the allocation strategy proposed in [7] by introducing the SC scheme [11] on both MA and RA algorithm, when considering the real time and non real time users respectively and we assume perfect knowledge of CSI at BS. The proposed MA-SC and RA-SC algorithm achieves maximum throughput with power constraint of real and non real time users, satisfying QoS requirement and proportional fairness of real and non real time users respectively. We also add some new constraints to [7], explained in sect. II to satisfy the requirement of SC theorem. (MA-SC) algorithm based on marginal utility of subcarrier, determines power required by real time users $\mathrm{P}_{\mathrm{rt}}^{*}$ by allowing first degraded real time user (far from BS) to look for the best subcarrier for him on the basis of channel gain then potential real time user (near to BS) is allowed to share the same subcarrier by transmitting small amount of power without deteriorating the performance of degraded user. (RA-SC) algorithm gives the throughput of non real time user by utilizing the remaining power $\mathrm{P}^{*}$ nrt and $\mathrm{SC}$ scheme as followed in MA-SC. In MA-SC, inclusion of potential user algorithm in fourth step increases the complexity to $\mathrm{O}\left(\mathrm{W}_{\mathrm{rt}} \mathrm{N}\right)$ from $\mathrm{O}(\mathrm{N})$ of $\mathrm{MA}$ algorithm [7], where Wrt is total number of real time users and $\mathrm{N}$ is total 
number of subcarriers and $\mathrm{N}>\mathrm{W}_{\mathrm{rt}}$. However, the overall complexity of MA-SC remain same as MA algorithm [7]. In the proposed RA-SC, the complexity remains the same as that of RA [7].

The layout of paper as follows: Sect. II describes optimization problem of bit and subcarrier allocation. Sect. III explains SC theorem. Sect. IV explains (MA-SC) and (RA-SC) algorithms. Sect. V presents simulation results. Finally, conclusions are drawn in Sect. VI.

\section{OPTIMIZATION PROBLEM}

The optimization problem of the subcarrier and bit allocation algorithm with inclusion of SC for both real time (degraded and potential) user and non real time (degraded and potential) is to maximize system throughput, ensuring the bit rate requirement of realtime users and the proportional fairness among non-realtime users subject to power constraint for real and non real time users.

Let us have $\mathrm{W}$ users that include Wrt realtime (degraded and potential) users and Wnrt non-realtime (degraded and potential) users with requirement of BER \& bit rate and without requirement of bit rate respectively. The complete set of realtime users and non-realtime users which contain both degraded and potential users are denoted by $\mathrm{U}^{*}{ }_{\mathrm{rt}}$ and $\mathrm{U}^{*}{ }_{\mathrm{nrt}}$ respectively. The downlink channel gain of the $\mathrm{k}_{\mathrm{th}}(1<\mathrm{k}<\mathrm{M})$ user on the $\mathrm{n}_{\text {th }}(1<\mathrm{n}<\mathrm{N})$ subcarrier is given by $\mathrm{h}^{*} \mathrm{kn}$.

The optimization scheme [1] is modified with increased constraints described below:

$$
R_{T}=\max _{a_{k n}^{*} \varepsilon[0,1] b_{k n}^{*} \varepsilon L} \sum_{k \varepsilon U_{n r t}^{*}} \sum_{n=1}^{N} a_{k n}^{*} b_{k n}^{*}
$$

The maximization is subjected to the constraints given below:

$$
\begin{aligned}
& \mathrm{C} 1: \sum_{k=1}^{K} a_{k n}^{*} \leq 2, \forall \mathrm{n} \\
& \mathrm{C} 2: R_{k}=R_{k}^{r e q}, \forall \mathrm{k} \mathcal{E} U_{r t}^{*} \\
& \mathrm{C} 3: R_{i} / \Omega_{i}=R_{j} / \Omega_{j}, \forall \mathrm{i}, \mathrm{j} \mathcal{E} U_{n r t}^{*} \\
& \mathrm{C} 4: \sum_{n \varepsilon S_{r t}^{*}} p_{n} \leq P_{T}, \sum_{n \varepsilon S_{n r t}^{*}} p w r_{n} \leq P_{n r t}^{*} \\
& \mathrm{C} 5: p_{n} \geq 0, p w r_{n} \geq 0 \\
& \mathrm{C} 6: \sum_{k=1}^{K} \beta_{k, n}^{\text {pot }} \leq 1, \sum_{k=1}^{K} \beta_{k, n}^{\text {deg }} \leq 1, \forall \mathrm{n} \\
& \mathrm{C} 7: \beta_{k, n}^{d e g} \beta_{k, n}^{p o t}=0, \forall \mathrm{n}, \forall \mathrm{k} \\
& \mathrm{C} 8: \sum_{k=1}^{K} \sum_{n \varepsilon S_{n r t}^{*}} a_{k n}^{*} p_{k n}^{*} \leq P_{n r t}^{*}
\end{aligned}
$$

Here, L denotes the set of natural numbers; C1 guarantees that each subcarrier can be shared by at most two user. The bit rate requirement $\mathrm{R}_{\text {req }}^{\mathrm{k}}$ of realtime users $\mathrm{k}$ is indicated by C2. One can achieve the proportional fairness for non-realtime users using C3 with rate weight of user $\mathrm{k}$ denoted by $\Omega_{\mathrm{k}}$ satisfies $\sum_{k \varepsilon U * n r t} \Omega \mathrm{k}=1$. In $\mathrm{C} 4$, the sum of power on each real and non real time subcarriers should be less than equal to $\mathrm{P}_{\mathrm{T}}$ and $\mathrm{P} *$ nrt respectively. $\mathrm{C} 5$ indicates power on each real and non real time subcarriers should be positive. C6 denotes subcarrier allocation indicator for the potential and degraded user. C7 explains that one user cannot be considered as potential and degraded on the same subcarrier. C8 is maximum power constraint for non real time users.

\section{SuPERPosition CODING SCHEME}

Superposition coding [11] allows at most two users to share each subcarrier for their transmission. Independent resultant information $\mathrm{S}$ corresponding to the degraded and potential user $S_{1}$ and $S_{2}$ respectively is transmitted to two or more receivers at the same time.

$$
\mathrm{S}=\mathrm{S}_{1}+\mathrm{S}_{2} / \mathrm{A}
$$

where A equal to 8 is ratio of adjusted power between the degraded and potential user signal. Single tx and $r x$ antenna is used. The signal received at receiver $\mathrm{Y}$ is decoded into $\mathrm{S}_{1}^{\wedge}$ and $\mathrm{S}_{2}$ (expressed below), which denotes the received signal corresponding to the degraded and potential user side respectively.

$$
\begin{gathered}
\mathrm{S}_{1}^{\wedge}=\mathrm{h}^{*}{ }_{\text {deg }} \mathrm{S}+\mathrm{Z}^{*}{ }_{\text {deg }} \\
\mathrm{S}_{2}{ }_{2}=\mathrm{h}^{*}{ }_{\text {pot }} \mathrm{S}+\mathrm{Z}^{*}{ }_{\text {pot }}
\end{gathered}
$$

where $\mathrm{h}^{*}{ }_{\text {deg }}$ and $\mathrm{h}{ }^{*}{ }_{\text {pot }}$ are channel gain, $\mathrm{Z}^{*}{ }_{\text {deg }}$ and $\mathrm{Z}^{*}{ }_{\text {pot }}$ are (AWGN) noise for degraded and potential user respectively.

The mechanism to decode the signal of degraded and potential user at receiver is similar to [11]. $\mathrm{U}^{*}$ pot 's signal is considered as a noise by $\mathrm{U}^{*}{ }_{\mathrm{deg}}$ and then $\mathrm{U}^{*}{ }_{\mathrm{deg}}$ decodes its own signal from $\mathrm{S}_{1}^{\wedge}$. Now the turn for the potential user to decode its own signal. Successive interference cancelation (SIC) is performed by $\mathrm{U}^{*}$ pot who has the best channel gain. This means that at potential user side, when $S_{1}$ is decoded, $U^{*}$ pot moves away from $\mathrm{S}_{2}$ to decode $\mathrm{S}_{2}$. Some practical simplification [11] is being done to reduce the complexity which are as follows:

1) Potential user can only transmit 2 bits using QPSK modulation, otherwise potential user $\mathrm{S}_{2}$ is not allowed to transmit any information.

2) In order to know that a particular user is potential or not on a specific subcarrier, a predetermined threshold given in Table I is used for comparison with user's SNR.

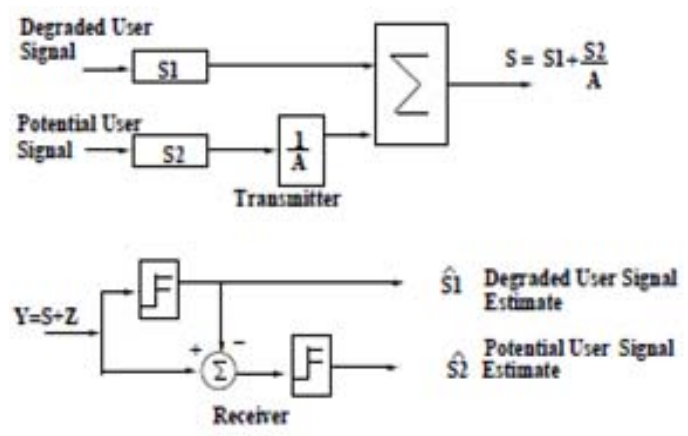

Fig. 1. Transmission and Reception Scheme for SC [9].

\section{The Subcarrier And Bit Allocation TeChNiQue}

In this section, we develop (MA-SC) and (RA-SC) algorithm in order to determine the power required by the real time user and throughput of non-real time user respectively, where real and non real time user consist of potential and degraded users. The total power available at the base station 
$\mathrm{P}_{\mathrm{T}}$ is utilized in such a way that power required by the real time (degraded and potential) user using MA-SC comes out to be $\mathrm{P}_{\mathrm{rt}}$. The remaining power $\mathrm{P}^{*}{ }_{\text {rrt }}$ is obtained by subtracting $\mathrm{P}_{\mathrm{rt}}$ from total power $\mathrm{P}_{\mathrm{T}}$ and remaining subcarrier is obtained as $\mathrm{S}^{*} \mathrm{nrt}=(1,2, \ldots . \mathrm{N})-\mathrm{S}^{*} \mathrm{rt}$. This remaining power and subcarrier is used in RA-SC for calculating the throughput of non real time (potential and degraded) users.

\section{A. Margin Adaptive Algorithm with Superposition Coding scheme}

The objective of (MA-SC) algorithm is to achieve minimization of total required power satisfying the requirement of bit rate for realtime (Potential and degraded) users and constraint $\sum_{n E S * r t} \mathrm{p}_{\mathrm{n}} \leq \mathrm{P}_{\mathrm{T}} . \mathrm{S}{ }_{\mathrm{rt}}$ is total subcarriers available to the real time users. In this algorithm, joint subcarrier $\mathrm{a}^{*}{ }_{\mathrm{kn}}$ and bit allocation $\mathrm{b}^{*}{ }_{\mathrm{kn}}$ is considered. MA-SC is based on the notion of marginal utility of subcarrier [7], which gives the maximum reduction in power, when subcarrier $\mathrm{n}$ is additionally allocated to user $\mathrm{k}$, which is denoted as $\Delta$ pwm $_{\mathrm{kn}}$. MA algorithm [7] is modified by introducing SC scheme [11]. The proposed modified algorithm is described below:

Input $\mathrm{S}^{*} \mathrm{rt}, \mathrm{P}_{\mathrm{T}}$ and Output $\mathrm{P}^{*} \mathrm{rt}$.

\section{Initialization}

a) set $\mathrm{a}^{*}{ }_{\mathrm{kn}=}=0, \mathrm{~b}{ }_{\mathrm{kn}}=0, \beta_{\mathrm{k}, \mathrm{n}}{ }^{\mathrm{deg}}=0$ and $\beta_{\mathrm{k}, \mathrm{n}}{ }^{\mathrm{pot}}=0$ for $\mathrm{k} \mathcal{E} \mathrm{U}^{*}{ }_{\mathrm{n}}$ and $\mathrm{n} \mathcal{E} \mathrm{S}^{*}{ }_{\mathrm{n}}$. Set $\mathrm{S}=\mathrm{S}^{*}{ }_{\mathrm{n}}, \mathrm{S}_{\mathrm{k}}=\varnothing$ and $\operatorname{Spot}=\varnothing, \forall \mathrm{k}$.

b) Power initialization: the amount of power on each real time subcarrier $S^{*}{ }_{n}$ is $p_{n}=P_{T} /\left|S_{n}^{*}\right|$, where $P_{T}$ is the total power available at the base station.

\section{2. for each $\mathrm{k} \mathcal{E} \mathrm{U}^{*}{ }_{\mathrm{n}}$}

a) Sort $\mathrm{h}^{*}{ }_{\mathrm{kn}}$ in ascending order

b) Find $n^{*}=\arg \max _{n} \varepsilon S h^{*}{ }_{k n}$

c) set $\mathrm{a}^{*} \mathrm{kn}^{*}=1, \beta_{\mathrm{k}, \mathrm{n}^{*}}{ }^{\operatorname{deg}}=1$ and $\mathrm{b}^{*}{ }_{\mathrm{kn}}{ }^{*}=\mathrm{R}_{\mathrm{k}}{ }^{\text {req }}, \mathrm{S}=\mathrm{S}-\{\mathrm{n} *\}$, $\mathrm{S}_{\mathrm{k}}=\mathrm{S}_{\mathrm{k}}+\{\mathrm{n} *\}$.

d) looking for potential user

i. From $b^{*}{ }_{k n} *$, determine the number of bits transmitted by user $\mathrm{k}$ on subcarrier $\mathrm{n}^{*}$, then obtain $\mathrm{SNR}_{\mathrm{deg}}{ }^{\mathrm{min}}$ from Table I

ii. Adjust transmit power $\mathrm{pn}^{*}$ to get $\mathrm{SNRk}, \mathrm{n}^{*}=\left(\mathrm{p}_{\mathrm{n}^{*}}\left(\mathrm{~h}^{*} \mathrm{kn}^{*}\right)^{2}\right) /\left(\sigma_{\mathrm{k}, \mathrm{n}^{*}}^{2}\right)$ equal to $\mathrm{SNR}_{\min }{ }^{\mathrm{deg}}$ from Table I.

iii. for each user $\mathrm{k}$ recompute the SNR

iv. call potential user finding algorithm described in section IV $(\mathrm{c})$.

\section{3. for each $\mathrm{k} \mathcal{E} \mathrm{U}^{*}{ }_{\mathrm{n}}$}

a) Find $n^{*}=\arg \max _{n} \varepsilon s h_{k n}^{*}$

b) Calculate marginal utility $\Delta \mathrm{pwm}_{\mathrm{k}, \mathrm{n}^{*}}$ using the approach explained in [7].

\section{While $\mathrm{S} \neq \varnothing$}

a) Find $\left(\mathrm{k}^{*}, \mathrm{n}^{*}\right)=\arg \max _{\mathrm{k}, \mathrm{n}} \varepsilon \mathrm{S} \Delta \mathrm{pwm}_{\mathrm{k}, \mathrm{n}}$

b) set $\mathrm{a}^{*}{ }_{\mathrm{k}^{*}, \mathrm{n}^{*}}=1, \beta_{\mathrm{k}^{*}, \mathrm{n}^{*}}{ }^{\operatorname{deg}}=1, \mathrm{~S}=\mathrm{S}-\{\mathrm{n} *\}, \mathrm{S}_{\mathrm{k}}=\mathrm{S}_{\mathrm{k}}+\{\mathrm{n} *\}$

c) Redistribute the required bits of user $\mathrm{k}^{*}$ to subcarrier set $\mathrm{S}_{\mathrm{k}}$.

d) looking for potential user as done in 2(d) of same algorithm with $\mathrm{k}$ is replaced by $\mathrm{k}^{*}$.

e) for all user $\mathrm{k}$ whose $\Delta$ pwmkn* using the approach explained in [7].

5. $\mathrm{a}^{*} \mathrm{kn}$ and $\mathrm{b}^{*} \mathrm{kn}$ is final subcarrier and bit allocarion indicators. The overall transmit power is given as

$$
P_{r t}^{*}=\sum_{k \varepsilon U_{r t}^{*}} \sum_{n \varepsilon s_{r t}^{*}} a_{k n}^{*} \frac{\Gamma \sigma^{2}}{h_{k n}^{*}}\left(2^{b_{k n}^{*}}-1\right)
$$

Explanation: The subcarrier and bit allocation indicators is initialized in first stage along with the separate allocation coefficients for both degraded $\beta_{\mathrm{k}, \mathrm{n}}{ }^{\mathrm{deg}}$ and potential user $\beta_{\mathrm{k}, \mathrm{n}}{ }^{\text {pot }}$. $\mathrm{S}$ have the knowledge of the unallocated subcarrier, $\mathrm{S}_{\mathrm{k}}$ and $\mathrm{S}_{\text {pot }}$ will depict the subcarrier set assigned to degraded users and potential users respectively. Also in first stage, equal amount of power $\mathrm{P}_{\mathrm{T}}$ is allocated on different subcarriers $\mathrm{S} * \mathrm{rt}$, where $|$.$| denotes the cardinality of a set.$

In the second stage, subcarrier which is not being utilized and also has the maximum channel gain is assigned to user at each iteration. These users are degraded real time users, who can transmit the total number of bits available to them by bit rate requirement on subcarrier recently found at each iteration. Then we look for the potential user who can transmit 2 bits on same subcarrier using the SC. To look for potential user, the no. of bits transmitted by degraded user and 2 bits by potential user is compared in the Table I and corresponding $\mathrm{SNR}_{\mathrm{deg}}{ }^{\min }$ is found. Then transmit power $\mathrm{p}_{\mathrm{n}^{*}}$ is compensated inorder to adjust

$\mathrm{SNR}_{\mathrm{k}, \mathrm{n}}{ }^{*}=\mathrm{p}_{\mathrm{n}} *\left(\mathrm{~h}_{\mathrm{k}, \mathrm{n}}^{*}\right)^{2} /\left(\sigma_{\mathrm{k}, \mathrm{n}}^{2}\right)$ equal to $\mathrm{SNR}_{\mathrm{deg}}$ min so that constraint $\sum_{n \varepsilon S * r t} p n$ is satisfied and then we recompute the SNR on the subcarrier $n^{*}$ for each user k. After that, we compare the SNR of all the potential users with the $\mathrm{SNR}_{\mathrm{pot}}$ min to decide that if a share allocation on the subcarrier $n^{*}$ is possible or not and if possible i.e potential user is found, then we add $n *$ to $S_{\text {pot. }}$ It is also important to note that one user cannot be considered as potential and degraded on the same subcarrier.

In the third stage, we calculate the marginal utility [7] of the unassigned subcarrier that are having the highest channel gain for each user. In following subcarrier selection stage, we would select only those subcarrier that are having largest marginal utility.

In the fourth stage, we jointly select an unallocated subcarrier and a particular user that are having the largest marginal utility, and we allocate this subcarrier to the selected user. Then we redistribute part of the required bits of the user to the recently added subcarrier. After this, we look for the potential user as done in step 2(d). There may be possibility that selected subcarrier is the best subcarrier for other users, then in that case the marginal utility of another unallocated subcarrier for those users are necessary to be calculated. Finally, we obtain subcarrier and bit allocation results and the overall power required to allocate all the subcarriers. In MA-SC, inclusion of potential user algorithm in fourth step increases the complexity to $\mathrm{O}(\mathrm{WrtN})$ from $\mathrm{O}(\mathrm{N})$ of MA algorithm [7]. However, the complexity of remaining step would remain same as MA [7], with no change in overall complexity.

\section{B. Rate Adaptive Algorithm with Superposition Coding Scheme}

RA-SC algorithm maximizes the total throughput of non-realtime (Potential and degraded) users by allowing both potential and degraded user to share the same subcarrier, subject to non real time power constraint $\mathrm{C} 8$ of optimization 
problem along with constraint $\sum_{n \varepsilon S * n r t} p w r_{\mathrm{n}} \leq \mathrm{P}^{*}{ }_{\text {nrt }}$ and user rate proportionality. In this algorithm, we separately perform subcarrier and bit allocation procedure. RA algorithm [7] is modified by introducing SC scheme [11]. Modified subcarrier allocation step including SC is given below:

Input: $\mathrm{S}^{*}{ }_{\text {nrt }}, \mathrm{P}^{*}{ }_{\text {nrt }}$ Output: $\mathrm{R}_{\mathrm{T}}$.

1. Initialisation

a) set $a^{*}{ }_{k n}=0, b^{*}{ }_{k n}=0, \beta_{k, n}{ }^{\operatorname{deg}}=0$ and $\beta_{k, n}{ }^{p o t}=0, b^{*}{ }_{k n}=, S_{k}=\varnothing$ Spot $=\varnothing$ and $\mathrm{R}_{\mathrm{k}}=\varnothing$, for $\mathrm{k} \mathcal{E} \mathrm{U}^{*}{ }_{\text {nrt }}$ and $\mathrm{n} \mathcal{E} \mathrm{S}^{*}{ }_{\text {nrt }}$. Set $\mathrm{S}=\mathrm{S}^{*}{ }_{\text {nrt }}$. Calculate $\mathrm{b}^{*}{ }_{\mathrm{kn}}$ as

$$
b_{k n}^{*}=\left\lfloor\log _{2}\left(1+\frac{h_{k n}^{*}}{\Gamma \sigma^{2}} \frac{P_{n r t}^{*}}{\left|S_{n r t}^{*}\right|}\right)\right\rfloor
$$

b) power initialization: the amount of power on each real time subcarrier $\mathrm{S}^{*}$ nrt is $\mathrm{pwrn}=\mathrm{P}^{*}{ }_{\mathrm{nrt}} /\left|\mathrm{S}^{*}{ }_{\text {nrt }}\right|$, where $\mathrm{P}^{*}{ }_{\text {nrt }}$ is remaining power available at the base station

2. While $\mathrm{S}_{\neq \varnothing}$

a) Find $\mathrm{k}^{*}=\arg \operatorname{mink}\left(\mathrm{R}_{\mathrm{k}} / \Omega_{\mathrm{k}}\right)$

b) Find $n^{*}=\arg \max b^{*}{ }_{k * n}$

c) set $\mathrm{a}^{*}{ }_{\mathrm{k}^{*} \mathrm{n}^{*}=1,} \quad \beta_{\mathrm{k}, \mathrm{n}}{ }^{\operatorname{deg}}=1 \quad$ and $\mathrm{S}=\mathrm{S}-\{\mathrm{n} *\}, \quad \mathrm{S}_{\mathrm{k}}=$ $\mathrm{S}_{\mathrm{k}^{*}}+\left\{\mathrm{n}^{*}\right\}, \mathrm{R}_{\mathrm{k}^{*}}=\mathrm{R}_{\mathrm{k}^{*}}+\mathrm{b}^{*} \mathrm{k}^{*} \mathrm{n}^{*}$.

d) looking for potential user as done in step 2(d) of MA-SC algorithm, with $\mathrm{k}$ and $\mathrm{p}_{\mathrm{n}}$ is replaced by $\mathrm{k}^{*}$ and pwrn respectively.

3. $\mathrm{a}_{\mathrm{kn}}$ is the total subcarrier allocation indicator, $\mathrm{S}_{\mathrm{k}}$ and $\mathrm{S}_{\mathrm{pot}}$ is the sucarrier set assigned to degraded and potential user respectively.

Bit allocation step using information from above as follows.

Inputs a* ${ }_{k n}, \mathrm{~S}_{\mathrm{k}}, \mathrm{S}_{\mathrm{pot}}$ and $\mathrm{P}{ }_{\text {nrt. }}$. Output $\mathrm{R}_{\mathrm{T}}$.

1. Initialisation

a) Set $b^{*}{ }_{k n}=0, R_{k}=0$, for $\mathrm{k} \mathcal{E} U^{*}{ }_{\text {nrt }}$ and $\mathrm{n} \mathcal{E} \mathrm{U}^{*}{ }_{\mathrm{nrt}}$ and $\mathrm{n} \mathcal{E} \mathrm{S}_{\mathrm{k}}$ Initalise $\mathrm{P}=0$.

$$
\Delta P_{k n}^{*}=\frac{\Gamma \sigma^{2}}{h_{k n}^{*}}\left(2^{b_{k n}^{*}}\right)
$$

b) Transmit 2 bits to each user $\mathrm{k}$ according to subcarrier allocation set Spot obtained from subcarrier allocation step. These users are potential users.

2. While (1)

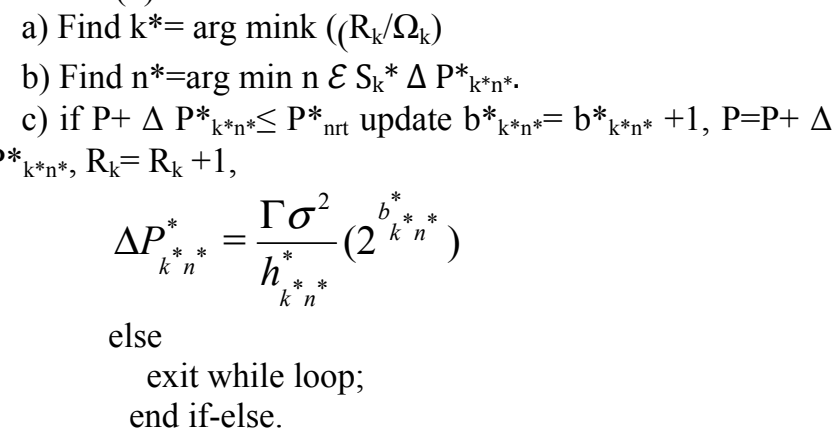

3. The final bit allocation of the $\mathrm{k}_{\mathrm{th}}$ user on the $\mathrm{n}_{\mathrm{th}}$ subcarrier is given as $b^{*}{ }_{k n}$. The overall throughput of non real time user is given by

$$
R_{T}=\sum_{k_{\varepsilon} U_{n r t}^{*}} \sum_{n \varepsilon S_{n r t}^{*}} a_{k n}^{*} b_{k n}^{*} .
$$

Explanation: The subcarrier allocation step is repeated up to the length of $\left|S^{*}{ }_{\text {nrt }}\right|$, where $|$.$| denotes the cardinality of a$ set and we initialize the subcarrier allocation indicator along with separate allocation coefficients for both degraded and potential user. $\mathrm{S}_{\mathrm{k}}$ and Spot are subcarrier set allocated to degraded and potential users respectively. $\mathrm{S}$ keeps the knowledge of the unallocated subcarriers. The remaining power $\mathrm{P}^{*}{ }_{\text {nrt }}$ is equally divided among the remaining subcarrier $\left|\mathrm{S}^{*}{ }_{\text {nrt }}\right|$. User rate proportionality is guaranteed by allowing that user to choose a subcarrier which has minimum normalized transmit rate $R_{k} / \Omega_{k}$. Now, selected user will choose that subcarrier which has highest transmit rate and these users are named as degraded users. Then we will look for potential user, who can transmit 2 bits on same subcarrier using SC. To look for potential user, we need to follow the same step as followed in 2(d) and 4(d) of (MA-SC) algorithm. One user cannot be considered as potential and degraded on the same subcarrier. After all the subcarriers in $\mathrm{S}^{*}{ }_{\text {nrt }}$ are assigned, we obtain the total subcarrier allocation indicator $\mathrm{a}^{*}{ }_{\mathrm{kn}}$ and the final assigned subcarrier set $\mathrm{S}_{\mathrm{k}}$ and Spot of degraded and potential users respectively. These three $\mathrm{a}_{\mathrm{kn}}$, $\mathrm{S}_{\mathrm{k}}$ and Spot are the input to bit allocation step.

The bit and corresponding power is allocated by bit allocation step on each subcarrier. Bit allocation step is similar to the subcarrier allocation step which assures to satisfy the user rate proportionality by allocating one bit to the user,who has least normalized rate $R_{k} / \Omega_{k}$ And we also allocate 2 bits to potential user according to subcarrier allocation set Spot. The next step allocate bits to degraded users.

In each iteration, the user will allocate additional bit to that subcarrier, which requires the least power. The bit allocation step ceases, when power allocated reaches the non real time power constraint $\mathrm{P}^{*}{ }_{\text {nrt }}$. With the addition of power initialization in 1st step, potential user algorithm in 2nd step of RA-SC (subcarrier allocation algorithm) and transmission of 2 bits to potential user of RA-SC (bit allocation algorithm) requires the complexity of $\mathrm{O}(\mathrm{N}), \mathrm{O}\left(\mathrm{W}_{\mathrm{nrt}} \mathrm{N}\right)$ and $\mathrm{O}\left(\mathrm{W}_{\mathrm{nrt}} \mathrm{N}\right)$ respectively. The overall complexity of RA-SC algorithm $\mathrm{O}\left(\mathrm{N}^{2}\right)$ would remain same as RA algorithm [7].

\section{Potential User Find Algorithm}

The algorithm for searching potential user [11] is described below:

- Set $\mathrm{m}=1$, Potential user=Not found,

- while $(\mathrm{m} \leq \mathrm{K})$ and (Potential User=Not found) do

- if SNRm,n* $\geq$ SNRpotmin then

- Potential User=Found and $m^{*}=\mathrm{m}$,

•b* $\mathrm{m}^{*} \mathrm{n}^{*}=$, Set $\mathrm{a}^{*}{ }_{\mathrm{m}^{*} \mathrm{n}^{*}}=1 \beta^{\mathrm{pot}}{ }_{\mathrm{m}^{*} \mathrm{n}^{*}}=1$

- $S_{p o t}\left(m^{*}\right)=S_{p o t}\left(m^{*}\right) \bigcup\left\{n^{*}\right\}$

- else

$$
\mathrm{m}=\mathrm{m}+1
$$

- end

TABLE I: THRESHOLd VALUE OF $\mathrm{SNR}_{\mathrm{K}}{ }^{\mathrm{MIN}}$ USING SC WITH SER $=10^{-3}$

\begin{tabular}{|l|l|l|l|}
\hline$R_{\text {deg }}^{*}$ & $R_{\text {pot }}^{*}$ & $S N R_{\text {deg }}^{\min }$ & $S N R_{\text {pot }}^{\min }$ \\
\hline 2 & 2 & $11.0 \mathrm{~dB}$ & $28.4 \mathrm{~dB}$ \\
\hline 4 & 2 & $18.3 \mathrm{~dB}$ & $35.4 \mathrm{~dB}$ \\
\hline 6 & 2 & $24.6 \mathrm{~dB}$ & $41.6 \mathrm{~dB}$ \\
\hline 8 & 2 & $30.7 \mathrm{~dB}$ & $47.1 \mathrm{~dB}$ \\
\hline 10 & 2 & $36.9 \mathrm{~dB}$ & $53.7 \mathrm{~dB}$ \\
\hline 12 & 2 & $42.9 \mathrm{~dB}$ & $59.8 \mathrm{~dB}$ \\
\hline 14 & 2 & $48.9 \mathrm{~dB}$ & $65.8 \mathrm{~dB}$ \\
\hline 16 & 2 & $54.9 \mathrm{~dB}$ & $71.8 \mathrm{~dB}$ \\
\hline
\end{tabular}


The Table I of [11] is expanded for $\mathrm{R}^{*}{ }_{\mathrm{deg}}=16$ bits, where $\mathrm{R}^{*}{ }_{\text {deg }}$ and $\mathrm{R}^{*}{ }_{\text {pot }}$ are bits transmitted by degraded and potential user respectively. We assume that same service is broadcasted to each user, which satisfy the following condition:

$\operatorname{SER}_{\text {degrad }}\left(\mathrm{S}_{1}\right) \leq 10^{-3}$, measured at degraded user side.

$\operatorname{SER}_{\text {potent }}\left(\mathrm{S}_{2}\right) \leq 10^{-3}$, measured at potential user side.

\section{Simulation Evaluation}

\section{A. Simulation Setup}

MATLAB tool has been utilized to evaluate the performance of the proposed MA-SC and RA-SC algorithm. Three-path rayleigh fading channel has been considered for simulation with an exponential power delay profile, whose variances are computed by $\mathrm{d}^{-\alpha}$ path-loss model for rayleigh fading channel, with $\alpha=3$. The total power $\mathrm{P}_{\mathrm{T}}$ at BS is $6.4 \mathrm{~W}$. Bandwidth assigned for OFDM system is $5 \mathrm{MHz}$, over this bandwidth BS will allocate 64 subcarriers. The average channel gain for both realtime (potential and degraded) users or non realtime (potential and degraded) users is chosen randomly within $(0.1,1)$. The bit error rate considered for each user is equal to $10^{-3}$ the SNR gap of OFDM system is about $5.48 \mathrm{~dB}$. AWGN's variance at each receiver is assumed to be equal, i.e $\sigma^{2}=1 \mathrm{~mW}$.

The number of real time users and non real time users are equal to 8 , and each user has the bit rate requirement $\mathrm{R}_{\mathrm{k}}{ }^{\text {req }}$ equal to 16 bits/OFDM symbol, and the rate weight of each non real time user satisfies: $\Omega_{\mathrm{k}}=1 / 8$, forall $\mathrm{k} \varepsilon \mathrm{U}^{*}{ }_{\text {nrt }}$. Averaging is done over 1000 network topologies over 100 OFDM symbols for each results.

\section{B. Simulation Results}

In Fig. $2, \mathrm{~W}_{\mathrm{rt}}=8$ and $\mathrm{R}_{\mathrm{k}}{ }^{\text {req }}=16$ bits/OFDM symbol forall $\mathrm{k} \varepsilon$ $\mathrm{U}^{*}{ }_{\mathrm{rt}}$. In proposed (MA-SC), power required to transmit a required number of bits for each real time user is reduced by $(57.14 \%)$, when 19 subcarriers are used and reduced by $(64.28 \%)$, when 21 subcarriers are used and reduced by (71\%), when 24 subcarriers are used and reduced by (30\%), when 32 subcarriers are used in comparison to MA [7], but now it requires to find the potential user at each iteration to transmit 2 bits to the user on the same subcarrier which had already been used by the degraded user. When we allocate 56 subcarriers, power required by real time user using MA and MA-SC algorithm comes out to be same, i.e., power become constant for both MA-SC and MA after 56 subcarriers have been allocated to real time users.

In Fig. $3, \mathrm{~W}_{\text {nrt }}=8$ and $\Omega_{\mathrm{k}}=1 / 8$, forall $\mathrm{k} \varepsilon \mathrm{U}^{*}{ }_{\text {nrt }}$. We notice that there is significant increment in the system capacity by using RA-SC. With RA [7], throughput at 16 subcarriers is zero but with RA-SC, it is 235 bits/OFDM symbol and when 24 subcarriers are used, throughput increases from 185 bits/OFDM symbol to 310 bits/OFDM symbol and when 32 subcarriers are used, throughput increases from 193 bits/OFDM symbol to 258 bits/OFDM symbols and throughput increases further in comparison to RA algorithm [7] for the remaining subcarriers.

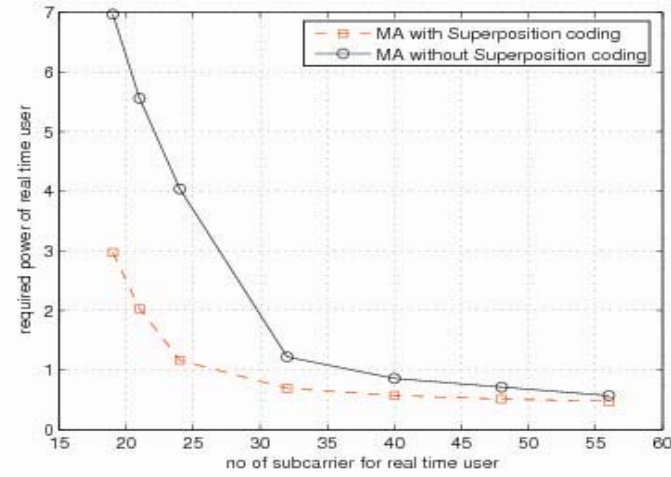

Fig. 2. Required power by real time user versus number of subcarriers for realtime user

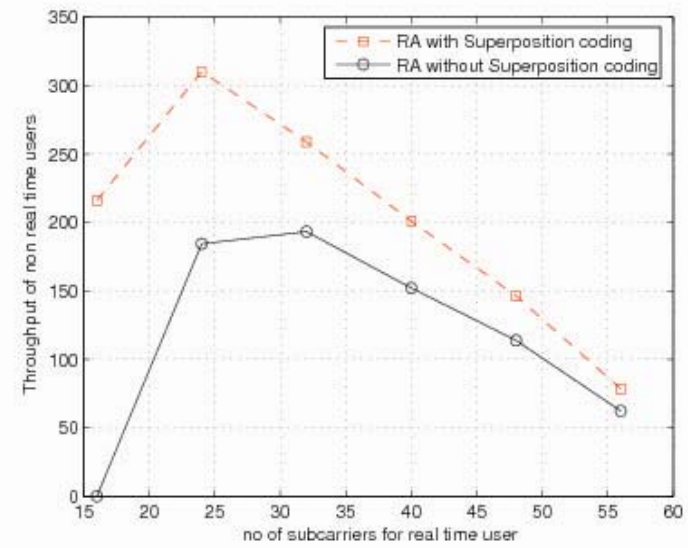

Fig. 3. Total throughput for non-realtime user v number of subcarriers used by real time user

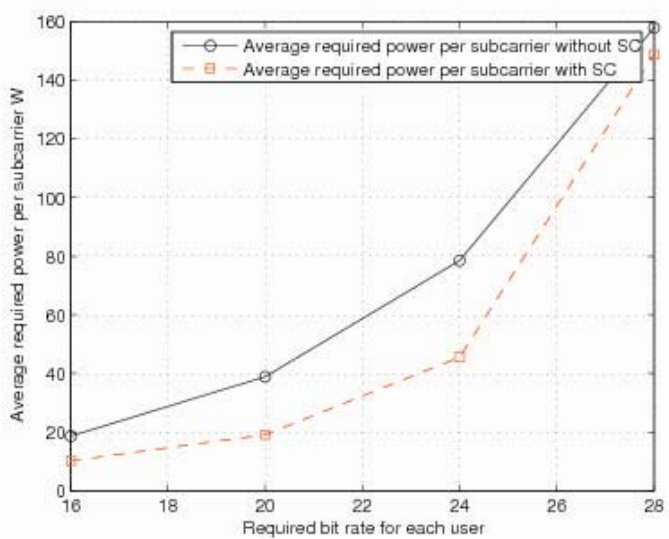

Fig. 4. Average required power per subcarrier vs required bit rate

In Fig. 4, average required power per subcarrier given as $\mathrm{P} *_{\mathrm{rt}} /\left(\mathrm{N} \sigma^{2}\right)$ is reduced as the power required by real time users is reduced. $\mathrm{W}_{\mathrm{rt}}$ varies from 4 to 8 . When 16 bits are used then average power per subcarrier is reduced by $26 \%$ and when 20 bits are taken then average power per subcarrier is reduced by $48.7 \%$ and when 24 bits are taken average power per subcarrier is reduced by $48.18 \%$ and it decreases further, when we go upto 28 bits. The bits required for Fig. 4 are taken in range from 16 to 28 bits/OFDM symbol for each user and elsewhere bits required have been taken equal to 16 bits/OFDM symbol. 


\section{CONCLUSION}

In this paper, we first study MA-SC algorithm, which jointly considers subcarrier and bit allocation for calculating the power required by real time (potential and degraded) users and then RA-SC algorithm, which separately allocate bit and subcarrier for maximizing the throughput of the non real time (potential and degraded) users utilizing the $\mathrm{P}^{*} \mathrm{nrt} \&$ Snrt. In both algorithms, each subcarrier is shared by at most two users as compared to MA and RA algorithm without SC scheme. System throughput is only dominated by throughput of non real time users, as throughput of the real time user is fixed and provided. The results show that our proposed algorithm performs better than MA and RA algorithm without SC [7]. In MA-SC, inclusion of potential user algorithm in fourth step increases the complexity to $\mathrm{O}\left(\mathrm{W}_{\text {nrt }} \mathrm{N}\right)$ from $\mathrm{O}(\mathrm{N})$ of MA algorithm [7]. The complexity of remaining step is same. The overall computational complexity of RA-SC algorithm is same as RA algorithm [7]. $\mathrm{SC}$ can be easily applied to any allocation algorithm for OFDM system as: whenever a subcarrier $\mathrm{n}$ is allocated to a user $k$, then we calculate $\mathrm{SNR}_{\mathrm{k}, \mathrm{n}}$ and compare this $\mathrm{SNR}_{\mathrm{k}, \mathrm{n}}$ to $\mathrm{SNR}_{\text {pot }}{ }^{\text {min }}$ from Table I so that we can have ac idea that how many bits can be transmitted and we treat this user as degraded. After this, we search for the potential user $\mathrm{u}$, who can use this remaining power for transmission of 2 more bits on the same subcarrier $\mathrm{n}$. Then we can declare user $\mathrm{k}$ as potential if its $\mathrm{SNR}_{\mathrm{k}, \mathrm{n}}$ is greater than $\mathrm{SNR}_{\mathrm{pot}}{ }^{\mathrm{min}}$ determined in Table I.

\section{ACKNOWLEDGMENT}

The authors are thankful to ABV-Indian Institute of Information Technology and Management Gwalior, India for extending the financial support for this paper.

\section{REFERENCES}

[1] J. G. Andrews, A. Ghosh and R. Muhamed, Fundamentals of WiMAX PrenticeHall,USA,2007.

[2] G. Yu, Z. Zhang, Y. Chen, J. Shi and P. Qiu, "A novel resource allocation algorithm for real time services in multiuser OFDM Systems," in IEEE 63 vehicular technology conference, vol. 3, Melbourne, Australia, May 7-10, 2006, pp. 1156-1160.

[3] C. Y. Wong, C. Y. Tsui, R. S. Cheng and K. B. Letaief, "A real time sub-carrier allocation scheme for multiple access downlink OFDM transmission," in Proc. IEEE VTC 1999-Fall, vol. 2, Sept. 1999, pp. 1124-1128.

[4] G. Zhang, "Sub carrier and bit allocation for real-time services in multi user OFDM systems," in Proc. IEEE ICC' 04, Vol. 5, Paris, France, June 20-24, 2004, pp. 2985-2989.

[5] D. Kivanc, G. Li, and H. Liu, "Computationally efficient bandwidth allocation and power control for OFDMA," IEEE Transactions on Wireless Communications, vol. 2, no. 6, pp. 1150-1158, November 2003.

[6] W. Wang, K. C. Hwang, K. B. Lee, and S. Bahk, ' Resource allocation for heterogeneous services in multiuser OFDM systems," in Proc. IEEE Globecom' 2004, vol. 6, Dallas, Texas, 2004, pp. 3478-3481.

[7] G. Yu, Z. Zhang, and P. Qiu, "Adaptive subcarrier and bit allocation in OFDMA systems supporting hetrogeneous services," Wireless Pers Communications, vol. 43, no. 4, pp. 1057-1070, Springer 2007.

[8] Q. Du, and X. Zhang, "Effective capacity of superposition coding based mobile multicast in wireless networks," in Proc. of IEEE ICC 2009, Dresden, Germany, June 14-18, 2009
[9] S. Najeh, H. Besbes, and A. Bouallegue, "A simple watermarking system," in Proc. ISCCSP 2004, Hammamet Tunisia, Mars, 2004, pp 531-534.

[10] S. Najeh, H. Besbes, and A. Bouallegue, "Greedy algorithm for dynamic resource allocation in downlink of OFDMA system,", in Proc. 2nd ISWCS 2005, Siena Italy September 5-7, 2005.

[11] H. Besbes, and S. Najeh, "A Simple Superposition Coding Scheme for Optimizing Resource Allocation in Downlink OFDMA Systems," Wireless Pers Communications, pp. 1-24, Springer 2010.

[12] H. Besbes, S. Najeh, A. Jdidi, and S. Smirani, "Resource allocation algorithm in downlink of OFDMA systems based on users classification and superposition coding," in Proc. SCS 2009, Djerba Tunisia, 2009, Nov.

[13] W. Rhee, and J. M. Cioffi, "'Increasing in capacity of multi user OFDM system using dynamic sub channel allocation". In Proc. IEEE Int. Vehicular Tech. Conf., vol. 2, Spring, 2000, pp. 1085-1089.

[14] Z. Shen, J. G. Andrews, and B. L. Evans, "Optimal power allocation in multi user OFDM systems," in Proc. IEEE GLOBECOM '03, vol. 1, San Francisco, CA, Dec. 1-5, 2003, pp. 337-341.

[15] I. C. Wong, Z. Shen, J. G. Andrews and B. L. Evans, "A low complexity algorithm for proportional resource allocation in OFDMA systems," in Proc. IEEE SIPS 2004, Austin, Texas, Oct. 13-15, 2004, pp. 1-6.

[16] G. Yu, Z. Zhang, Y. Chen, P. Cheng and P. Qiu, "Sub carrier and bit allocation for OFDMA systems with proportional fairness," in IEEE WCNC 2006, vol. 3, Las Vegas, USA, Apr. 3-6, 2006, pp. 1717-1722.

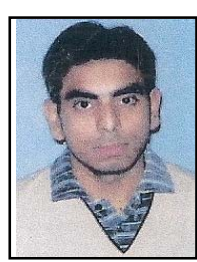

Karan Nathwani received the B.Tech degree in Electronics \& Communication from UPTU University in 2009 and M.Tech. degree in Digital Communication from ABV-IIITM Gwalior in 2011. He is now a Ph. D candidate in Electronics department in IIT Kanpur. His research interests include speech processing, wireless communication etc.

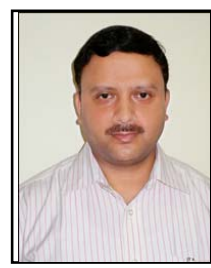

Dr. Aditya Trivedi is a Professor in the ICT Department of ABV-Indian Institute of Information Technology and Management, Gwalior, India. He received his bachelor degree (with distinction) in Electronics Engineering from the Jiwaji University and M.Tech. degree (Communication Systems) from Indian Institute of Technology (IIT), Kanpur. He obtained his doctorate $(\mathrm{PhD})$ from IIT Roorkee in the area of Wireless Communication Engineering.

His teaching and research interest include Digital communication, CDMA systems, Signal processing, and Networking. He is a fellow of the Institution of Electronics and Telecommunication Engineers (IETE) and a member of Institution of Electrical and Electronics Engineers (IEEE),USA. He has published more than 50 papers in various prestigious International/National journals and conferences. In 2007, he was given the IETE's K.S. Krishnan Memorial Award for best system oriented paper.

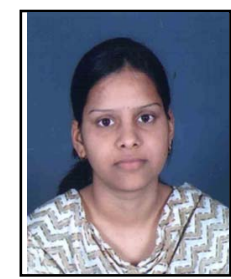

Kalpana Goyal received the B.E. degree in Electronics and Communication Engineering from Rajiv Gandhi Proudyogiki Vishwavidyalaya, Bhopal in 2008 and M.Tech. degree in Digital Communication from ABV-Indian Institute of Information Technology and Management, Gwalior, India in 2011.

She is now an Assistant Professor in Maulana Azad National Institute of Technology, Bhopal, India. Her research interests include MIMO OFDM systems, cross layer design, wireless communications etc. 